\title{
COMPOSIÇÃO FLORÍSTICA ARBÓREA EM RESERVA EXTRATIVISTA NO AMAPÁ
}

\author{
João da Luz Freitas* \\ Raullyan Borja Lima e Silva* \\ Francisco de Oliveira Cruz Junior*** \\ Patrick de Castro Cantuária**** \\ Tonny David Santiago Medeiros ${ }^{* * * * *}$ \\ Erick Silva dos Santos ${ }^{* * * * * *}$
}

RESUMO: O estudo avaliou a composição florística e a diversidade da vegetação do trecho denominado Alto Cajari, na Reserva Extrativista do Rio Cajari, abrangendo os municípios de Mazagão, Laranjal do Jari e Vitória do Jari no estado do Amapá. Foram alocadas 27 parcelas de $20 \times 100 \mathrm{~m}$, totalizando uma área amostrada de 5,4 hectares. Em cada parcela foi realizada a identificação e contagem do número de indivíduos com DAP $\geq 9 \mathrm{~cm}$.As tipologias vegetais encontradas no Alto Cajari são predominantemente dos ecossistemas de floresta ombrófila densa e savana amazônica. Nas 27 parcelas foram amostrados 437 indivíduos, 34 famílias e 74 espécies. Fabaceae (15 espécies), Anacardiaceae (5), Lauraceae e Myrtaceae (4) se sobressaíram em riqueza de espécies. As espécies que mais se destacaram foram Cecropia palmata Willd. (28), Byrsonima crassifolia (L.) Kunth (22), Himatanthus articulatus (Vahl) Woodson (21), Spondias mombin L. (19), Tabebuia aurea (Silva Manso) Benth.\& Hook. f ex S. Moore (18) e Trattinnickia rboifolia Willd. (18).

PALAVRAS-CHAVE: Extrativismo vegetal; Amazônia; Florística; Unidade de conservação.

Doutor em Ciências Agrárias, Pesquisador do Instituto de Pesquisas Científicas e Tecnológicas do Estado do Amapá (IEPA), Brasil. Email: jfreitas.ap@gmail.com

** Doutor em Ciências Socioambiental, Pesquisador do Instituto de Pesquisas Científicas e Tecnológicas do Estado do Amapá (IEPA), Brasil.

*** Mestre em Biodiversidade Tropical, Pesquisador do Instituto de Pesquisas Científicas e Tecnológicas do Estado do Amapá (IEPA), Brasil.

***** Doutor em Biodiversidade e Tecnologia, Pesquisador do Instituto de Pesquisas Científicas e Tecnológicas do Estado do Amapá (IEPA), Brasil.

****** Mestre em Botânica, Pesquisador do Instituto de Pesquisas Científicas e Tecnológicas do Estado do Amapá (IEPA), Brasil. Mestre em Direito Ambiental e Políticas Públicas, Pesquisador do Instituto de Pesquisas Científicas e Tecnológicas do Estado do Amapá (IEPA), Brasil 


\title{
ARBOREAL FLORIST COMPOSITION IN AN EXTRACTIVIST RESERVE AREA IN AMAPÁ, BRAZIL
}

\begin{abstract}
Current paper assessed the florist composition and vegetation diversity of a stretch of forest called Alto Cajari, within the Extrativist Reserve of the River Cajari, in the municipalities of Mazagão, Laranjal do Jari and Vitória do Jari, in the state of Amapá, Brazil. Twenty-seven $20 \times 100$ m plots, total area of 5.4 hectares, were investigated. Identification and counts of specimens with DAP $\geq 9 \mathrm{~cm}$. Vegetal typologies in the Alto Cajari predominantly hail from the ecosystems of dense ombrophilous forest and Amazon savannah. The 27 plots comprised 437 specimens, 34 families and 74 species. Fabaceae (15 species), Anacardiaceae (5), Lauraceae and Myrtaceae (4) were highlighted in species richness. The most underscored species were Cecropia palmata Willd. (28), Byrsonima crassifolia (L.) Kunth (22), Himatanthus articulatus (Vahl) Woodson (21), Spondias mombin L. (19), Tabebuia aurea (Silva Manso) Benth.\& Hook. f ex S. Moore (18) and Trattinnickia rboifolia Willd. (18).
\end{abstract}

KEY WORDS: Vegetal extractivism; Amazon; Floristics; Conservation unit.

\section{INTRODUÇÃO}

A utilização e a conservação dos recursos naturais existentes, nos ecossistemas naturais e artificiais, estão condicionadas ao conhecimento e estudo dos componentes que formam a biodiversidade desses ecossistemas. Inserido entre os elementos formadores dos ecossistemas encontra-se a vegetação, da qual, o uso adequado consiste fundamentalmente na preservação e manutenção dos demais recursos naturais.

Estudos para a estimativa sobre o número de espécies existentes na Terra indicam que o ser humano compartilha o planeta com outros 8,7 milhões de seres diferentes, sendo que até o momento estima-se que já foram catalogadas em torno de 1,2 milhões de espécies, significa dizer que 7,5 milhões continuam desconhecidas pela ciência (MORA et al., 2011).

No entanto, Wilson (1997) estima que existam entre 5 e 30 milhões de espécies de organismos vivos, embora o número atual de espécies descritas seja de 1,4 milhão, sendo 320.000 de plantas vasculares e briófitas. Segundo estimativas do 
SiBBr (2016) e Flora do Brasil 2020 em construção (2016), o Brasil possui expressiva biodiversidade com valores acima de 40.000 espécies de plantas, o que em termo de Amazônia, maior bioma do país, calcula-se que este possui 2.500 espécies de árvores e 30 mil espécies de plantas (MMA, 2016).

Estudos de caracterização da vegetação são etapas fundamentais para o entendimento do ecossistema e do planejamento das atividades de manejo florestal acerca da composição da floresta a ser manejada (SANTOS et al., 2013; FREITAS et al., 2011). Segundo Higuchi, Santos e Jardim (1982), essa avaliação é feita por meio de inventários florestais, os quais qualificam e quantificam os recursos referentes às espécies vegetais ocorrentes com relação à tomada de decisão referente ao uso do solo.

Para Freitas (2008), a aplicação de técnicas adequadas ao uso dos recursos florestais de um ecossistema, passa, sobretudo, pelo conhecimento dos componentes formadores do mesmo e suas interações. Para tanto várias técnicas de levantamento da flora e fauna são amplamente conhecidas. No caso específico da flora uma das ferramentas mais utilizadas é o estudo da composição florística ou inventário florestal, que é a parte da ciência florestal que visa avaliar as variáveis qualitativas e quantitativas da floresta e suas inter-relações. Assim como dinâmicas de crescimento e sucessão florestal, servindo de base para a formulação de planos de utilização de recursos madeireiro e não madeireiros; manejo integrado da floresta, além de embasar propostas de desenvolvimento e política florestal.

No Amapá, de acordo com GEA (2009), existem 19 Unidades de Conservação (UC), destacando-se a Reserva Extrativista (Resex) do rio Cajari. A Resex foi criada em 1990 pelo decreto-lei 99.145 de 12 de março de 1990, possuindo uma área de 532.397,20 ha, apresenta obstáculos como as demais, quanto à identificação das espécies e suas potencialidades e gestão, demonstrado pelo fato de não possuir plano de manejo. Apesar do aumento de pesquisas no Estado do Amapá, relacionado ao esforço de coleta botânica e de inventários florestais, muitas questões sobre a composição e estrutura de suas vegetações permanecem escassas e fragmentadas.

Atualmente, poucos trabalhos podem ser referenciados quanto às composições florísticas dos ecossistemas formadores do Estado, no que diz respeito à diversidade, composição e estrutura vegetacional. Dessa forma, o estudo teve 
como objetivo analisar a estrutura e a composição florística de fragmentos florestais de terra firme e cerrado em trecho denominado Alto Cajari, na Resex do rio Cajari, entre os municípios de Mazagão, Laranjal do Jari e Vitória do Jari, Estado do Amapá.

\section{MATERIAL E MÉTODOS}

\section{1 ÁREA DE ESTUDO}

A Resex do rio Cajari está localizada na região Sul do Estado do Amapá, possuindo 532.397,20 ha que abrangem os municípios de Mazagão, Laranjal do Jari e Vitória do Jari. Nessa região, 720 famílias, distribuídas em 56 comunidades, sobrevivem da extração de castanhadobrasil (Bertholletia excelsa Bonpl.) e de açaizais nativos (Euterpe oleracea Mart.), pesca, atividade agrícola e outros recursos florestais não madeireiros (Figura 1). O acesso às suas comunidades é possibilitado por meio fluvial pelo rio Amazonas ou via terrestre pela rodovia BR156 que liga os municípios de Macapá e Laranjal do Jari (BENJAMIN, 2004; DRUMMOND; DIAS; BRITO, 2008; ESQUERDO; MARQUES; RANGEL, 2016). 

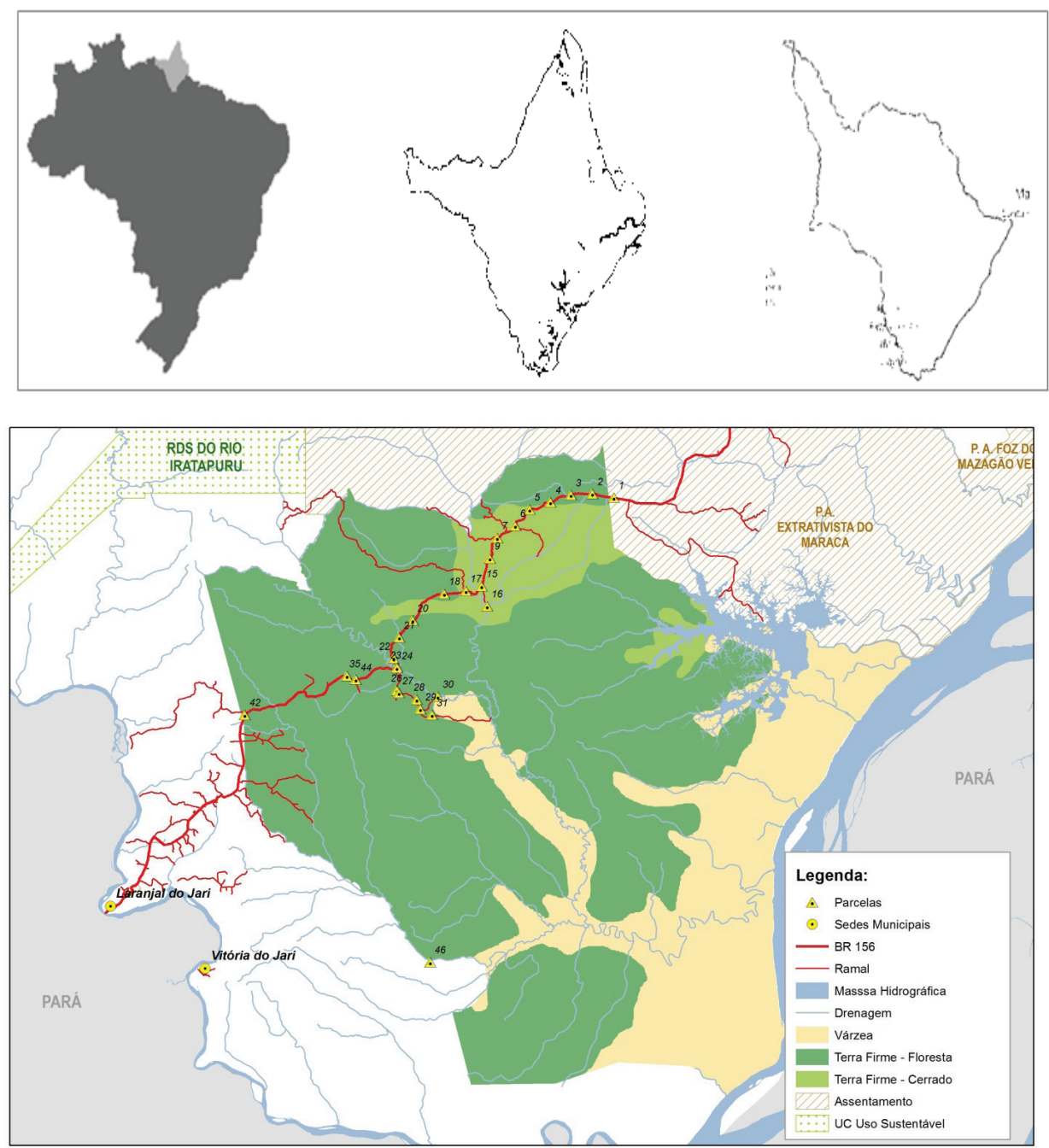

Figura 1. Localização da área de estudo e parcelas lançadas no trecho Alto Cajari, Resex do rio Cajari, municípios de Mazagão, Laranjal do Jari e Vitória do Jari, Estado do Amapá

A região apresenta alta pluviosidade, com precipitação anual que varia entre $2.300 \mathrm{~mm}$ e $2.400 \mathrm{~mm}$, com concentração dos maiores índices nos meses de fevereiro, março e abril. De acordo com a classificação de Köppen, o clima é do tipo Af na região próxima ao canal do norte (rio Amazonas) e Am no centro/norte da Resex do rio Cajari (ALVES, 1995).

A temperatura apresenta variação entre $16{ }^{\circ} \mathrm{C}$ e $38{ }^{\circ} \mathrm{C}$ e umidade relativa 
do ar com média anual de $85 \%$. Os solos da Resex podem ser considerados de textura média a argilosa e com baixa fertilidade, concreções e suscetível à erosão. Com relação à geologia e à geomorfologia, estão situadas na plataforma amazônica, formada em sua maior parte por sedimentos cenozoicos, pouco consolidados que revestem rochas sedimentares paleozoicas (ALVES, 1995).

A Resex do rio Cajari apresenta áreas com floresta ombrófila densa (terra firme), savana amazônica (cerrado) e várzea, destacando as espécies acapu (Vouacapoua americanaAubl.), açaizeiro (Euterpe oleracea), maçaranduba (Manilkara buberi (Ducke) Standl.), angelim-rajado, castanha-do-brasil (Bertholletia excelsa), (Zygia racemosa (Ducke) Barneby\& J.W. Grimes) e pau d'arco (Tabebuia serratifolia (Vahl) G. Nicholson). O relevo apresentado por essas florestas é denso de baixos platôs e densas submontana (DRUMMOND; DIAS; BRITO, 2008).

\subsection{PROCEDIMENTOS METODOLÓGICOS}

A metodologia empregada consistiu na utilização de amostragem sistemática (PELLICO NETO; BRENA, 1993; QUEIROZ, 1998). Durante 11 dias consecutivos em julho de 2013, foram alocadas 27 parcelas de $2.000 \mathrm{~m}^{2}$ (20 m x $\left.100 \mathrm{~m}\right)$, equidistantes entre si 3 quilômetros e distantes 10 metros da margem da rodovia e ramais de acesso, perfazendo um total de 5,4 hectares de área amostrada nos ambientes de floresta ombrófila densa (terra firme) e savana amazônica (cerrado).

As parcelas foram dispostas sistematicamente em subparcelas de $10 \mathrm{~m}$ x 20 m utilizando-se um retângulo formado com quatro piquetes de $2 \mathrm{~m}$. Essas recomendações estão de acordo com o proposto por Higuchi, Santos e Jardim. (1982), quanto a maior medida a ser sempre posicionada no sentido paralelo aos trechos de acesso (rodovia, estrada, rios e igarapés).

Os dados foram obtidos a partir da identificação e mensuração dos indivíduos arbóreos com diâmetro a altura do peito (CAP) a 1,30 m do solo $\geq 9,5$ $\mathrm{m}$. Estimaram-se as alturas comercial e total a partir de régua graduada de 6 metros de comprimento, posicionada junto ao indivíduo mensurado. Tais dados foram tabulados no software Microsoft Excel 2010.

A identificação taxonômica das espécies e redação dos nomes dos táxons 
foi realizada com base em Kew Monocot World Checklist (2012), Missouri Botanical Garden Tropicos (2012) e em "The Plant List". Já para a abreviatura dos nomes dos autores, seguiu-se o proposto por Brummitt; Powell (1992). Os nomes das famílias foram atualizados de acordo com o sistema proposto pelo APG III (2009). As grafias dos nomes das espécies, gêneros e famílias botânicas foram atualizadas mediante consulta no site Missouri Botanical Garden (MOBOT, 2015).

Foram calculados os parâmetros fitossociológicos para cada espécie com vistas à caracterização da estrutura da área amostrada (MUELLER-DOMBOIS; ELLENBERG, 1974), sendo determinados os seguintes parâmetros: Área basal (AB), Frequência Absoluta (FA), Frequência Relativa (FR), Densidade Absoluta (DA), Densidade Relativa (DR), Dominância Absoluta (DoA), Dominância Relativa (DoR), Índice de Valor de Importância (IVI) e Índice de Valor de Cobertura (VC) pelo software FITOPAC 1 (SHEPHERD, 1995).

\section{RESULTADOS E DISCUSSÃO}

\subsection{DISTRIBUIÇÃO DIAMÉTRICA DOS INDIVÍDUOS}

A composição florística inventariada registrou 437 indivíduos arbóreos, mensurados a partir do DAP $\geq 9,5 \mathrm{~cm}$. A área basal total foi de $26.238 \mathrm{~m}^{2}$ e o diâmetro médio $23.540 \mathrm{~cm}^{2}$. Os indivíduos foram distribuídos em classes de diâmetro para obtenção da altura comercial média (Tabela 1).

Tabela 1. Alturas médias comerciais por classe de DAP das árvores medidas no inventário florestal do trecho Alto Cajari, Resex do rio Cajari, Amapá

(Continua)

\begin{tabular}{ccccc}
\hline $\begin{array}{c}\text { Classes de } \\
\text { diâmetro }(\mathbf{c m})\end{array}$ & $\begin{array}{c}\text { Limites de DAP } \\
(\mathbf{c m})\end{array}$ & $\begin{array}{c}\text { Ponto } \\
\text { médio }\end{array}$ & Altura média $(\mathbf{m})$ & No indivíduos $^{\text {(m) }}$ \\
\hline 01 & $9,5-19,4$ & 14,4 & 4,9 & 248 \\
02 & $19,5-29,4$ & 24,4 & 6,6 & 98 \\
03 & $29,5-39,4$ & 34,4 & 7,9 & 46 \\
04 & $39,5-49,4$ & 44,4 & 9,0 & 13 \\
\hline
\end{tabular}


Conclusão

\begin{tabular}{ccccc}
\hline $\begin{array}{c}\text { Classes de } \\
\text { diâmetro }(\mathbf{c m})\end{array}$ & $\begin{array}{c}\text { Limites de DAP } \\
(\mathbf{c m})\end{array}$ & $\begin{array}{c}\text { Ponto } \\
\text { médio }\end{array}$ & Altura média $(\mathbf{m})$ & $\mathbf{N}^{\mathbf{0}}$ indivíduos \\
\hline 05 & $49,5-59,4$ & 54,4 & 11,4 & 14 \\
06 & $59,5-69,4$ & 64,4 & 10,9 & 14 \\
07 & Acima de 69,5 & 95 & 20,0 & 4 \\
\hline $\begin{array}{l}\text { Total de árvores } \\
\text { mensuradas }\end{array}$ & & & & 437 \\
\hline
\end{tabular}

A distribuição do número de indivíduos por classe de diâmetro segue uma função clássica de distribuição normal negativa ("J" invertido), apresentando uma concentração elevada de indivíduos nas classes menores de DAP e baixa nas classes maiores. No Alto Cajari, apenas quatro indivíduos apresentaram diâmetros acima de $69,5 \mathrm{~cm}$ (Figura 2).

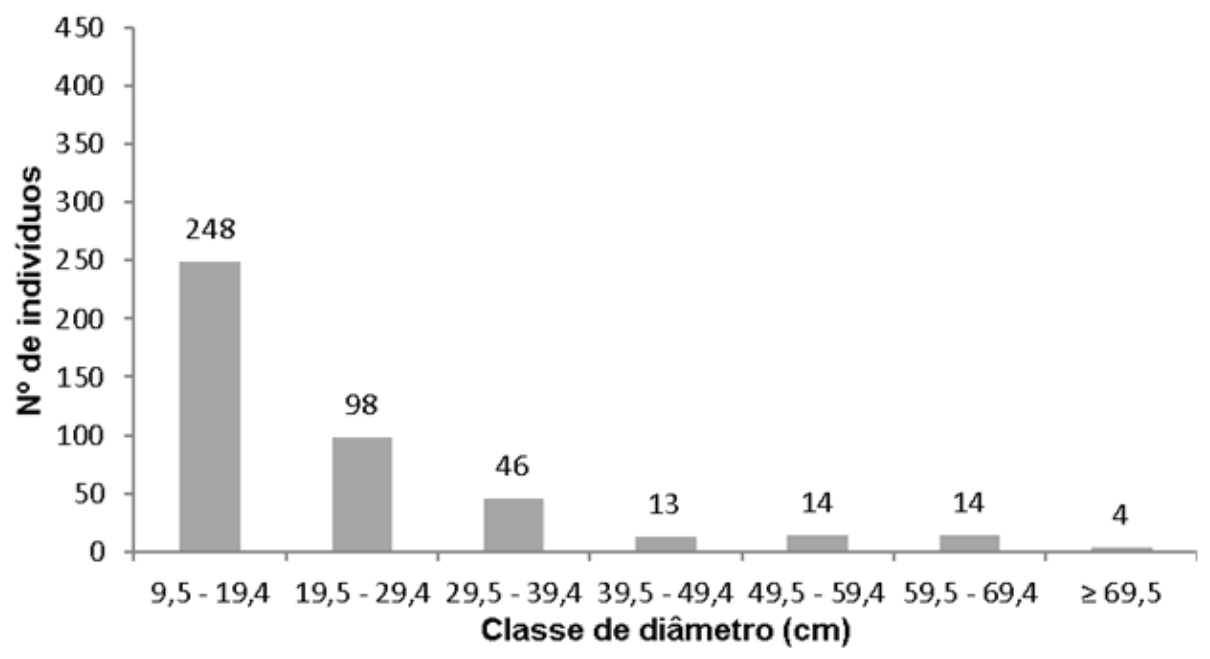

Figura 2. Distribuição do número de indivíduos por classe de diâmetro para as áreas amostradas do trecho Alto Cajari, Resex do rio Cajari, Amapá

A distribuição diamétrica na forma de "J" invertido parece ser uma característica das florestas tropicais amazônicas, sendo observados inúmeros estudos realizados na Amazônia (JARDIM, 1995; IVANAUSKAS; MONTEIRO; RODRIGUES, 
2004; SANTOS; JARDIM, 2006; FREITAS, 2008; GONÇALVES; SANTOS, 2008; APARÍCIO, 2011; SANTOS et al., 2013).

A intensidade amostral significativa é essencial para o real conhecimento da riqueza vegetal da área estudada, sendo uma relação do incremento da área amostrada e o número de espécies acumuladas (AMARAL; MATOS; LIMA, 2000), de maneira que essa não interfira negativamente nos resultados. Assim, ao se analisar a curva cumulativa, observa-se que há tendência de sua estabilização na parcela 27, indicando que os 5,4 hectares foram suficientes para amostrar a diversidade da maioria das espécies arbóreas com DAP $\geq 9,5 \mathrm{~cm}$ (Figura 3).

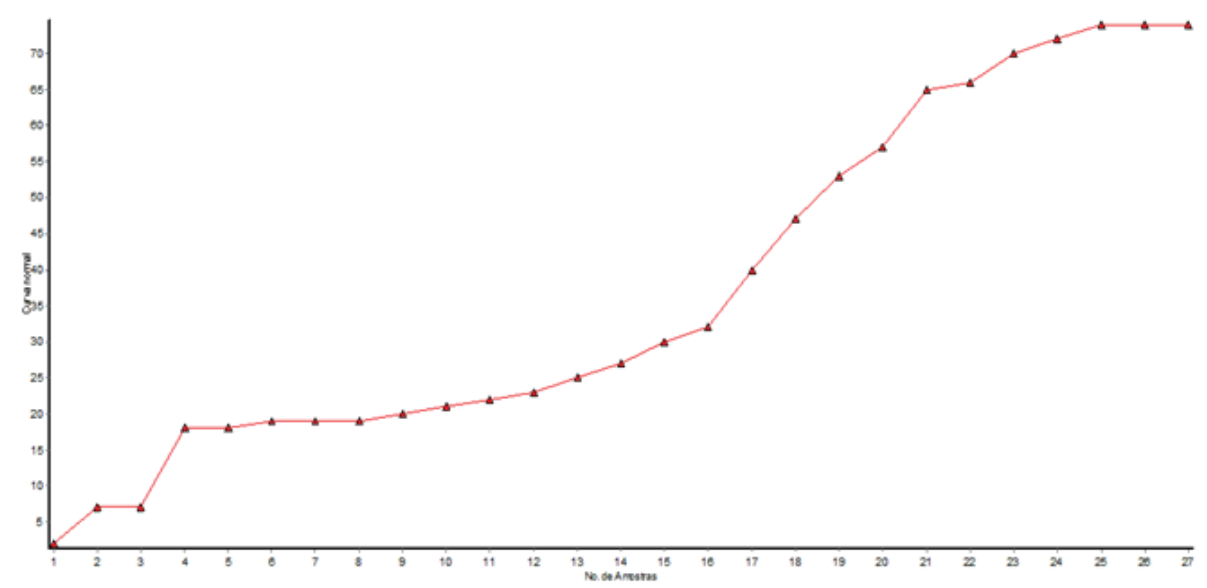

Figura 3. Curva cumulativa das espécies amostradas em 5,4 ha de vegetação no trecho Alto Cajari, Resex do rio Cajari, Amapá.

\subsection{ESPÉCIES E GÊNEROS}

Os 437 indivíduos inventariados pertencem a 34 famílias e 74 espécies, apresentando distribuição espacial agregada. Fabaceae, Anacardiaceae, Lauraceae e Myrtaceae contribuíram com $41,42 \%$ do número total de indivíduos e maior riqueza florística. Os gêneros mais representativos foram Apeiba, Myrcia e Saccoglottis (2 espécies), tendo os demais representados por apenas uma espécie (Tabela 2). 
Tabela 2. Parâmetros fitossociológicos das famílias e espécies amostradas em 5,4 ha de vegetação no trecho Alto Cajari, Resex do rio Cajari, Amapá

(Continua)

\begin{tabular}{|c|c|c|c|c|c|c|c|c|}
\hline Família & Espécies & NI & DA & DR & FA & FR & DoA & IVI \\
\hline Uticaceae & Cecropia palmata Willd. & 28 & 5.2 & 6.41 & 25.93 & 3.54 & 0.15 & 13.03 \\
\hline Malpighiaceae & $\begin{array}{c}\text { Byrsonima crassifolia (L.) } \\
\text { Rich. }\end{array}$ & 22 & 4.1 & 5.03 & 33.33 & 4.55 & 0.09 & 11.53 \\
\hline Apocynaceae & $\begin{array}{l}\text { Himathanthus articulata } \\
\text { (Vahl.) Wood. }\end{array}$ & 21 & 3.9 & 4.81 & 37.04 & 5.05 & 0.1 & 12.02 \\
\hline Anacardiaceae & Spondias mombin $\mathrm{L}$. & 19 & 3.5 & 4.35 & 7.41 & 1.01 & 0.26 & 10.75 \\
\hline Burseraceae & Trattinickia rboifolia Willd. & 18 & 3.3 & 4.12 & 29.63 & 4.04 & 0.18 & 11.95 \\
\hline Bignoniaceae & $\begin{array}{l}\text { Tabebuia aurea (Silva Manso) } \\
\text { Benth. \&Hook.f. exS.Moore }\end{array}$ & 18 & 3.3 & 4.12 & 25.93 & 3.54 & 0.09 & 9.60 \\
\hline Fabaceae & $\begin{array}{l}\text { Triplarisweigeltiana (Rchb.) } \\
\text { Kuntze }\end{array}$ & 17 & 3.1 & 3.89 & 11.11 & 1.52 & 0.11 & 7.62 \\
\hline Anacardiaceae & Anacardiumoccidentale $\mathrm{L}$. & 17 & 3.1 & 3.89 & 14.81 & 2.02 & 0.08 & 7.58 \\
\hline Fabaceae & Ingasp & 14 & 2.6 & 3.2 & 25.93 & 3.54 & 0.12 & 9.24 \\
\hline Myrtaceae & Myrciabracteata (Rich.) D.C. & 13 & 2.4 & 2.97 & 29.63 & 4.04 & 0.08 & 8.62 \\
\hline Bignoniaceae & $\begin{array}{c}\text { Jacarandacopaia (Aubl.) } \\
\text { D.Don }\end{array}$ & 13 & 2.4 & 2.97 & 18.52 & 2.53 & 0.11 & 7.67 \\
\hline Hypericaceae & $\begin{array}{l}\text { Vismia guianensis (Aubl.) } \\
\text { Pers. }\end{array}$ & 13 & 2.4 & 2.97 & 22.22 & 3.03 & 0.05 & 7.08 \\
\hline Dilleniaceae & Curatella americana $\mathrm{L}$. & 11 & 2 & 2.52 & 11.11 & 1.52 & 0.06 & 5.25 \\
\hline Fabaceae & $\begin{array}{c}\text { Dipteryxodorata (Aubl.) } \\
\text { Willd }\end{array}$ & 10 & 1.9 & 2.29 & 11.11 & 1.52 & 0.29 & 9.79 \\
\hline Fabaceae & PlathymeniafoliolosaBenth. & 10 & 1.9 & 2.29 & 14.81 & 2.02 & 0.05 & 5.26 \\
\hline Araliaceae & $\begin{array}{l}\text { Scheffleramorototoni (Aubl.) } \\
\text { Maguire, Steyerm. \&Frodin }\end{array}$ & 9 & 1.7 & 2.06 & 18.52 & 2.53 & 0.08 & 6.30 \\
\hline Fabaceae & BowdichiavirgilioidesKunth & 9 & 1.7 & 2.06 & 14.81 & 2.02 & 0.04 & 4.82 \\
\hline Anacardiaceae & Mangifera indica $\mathrm{L}$. & 8 & 1.5 & 1.83 & 11.11 & 1.52 & 0.13 & 5.94 \\
\hline
\end{tabular}


(Continua)

\begin{tabular}{|c|c|c|c|c|c|c|c|c|}
\hline Família & Espécies & NI & DA & DR & FA & FR & DoA & IVI \\
\hline Myrtaceae & $\begin{array}{l}\text { Syzygiummalaccense (L.) } \\
\text { Merr\& L.M. Perry }\end{array}$ & 8 & 1.5 & 1.83 & 7.41 & 1.01 & 0.12 & 5.39 \\
\hline Simaroubaceae & Simarouba amara Aubl. & 8 & 1.5 & 1.83 & 14.81 & 2.02 & 0.04 & 4.62 \\
\hline Lauraceae & $\begin{array}{l}\text { Licaria brasiliensis (Nees) } \\
\text { Kosterm. }\end{array}$ & 8 & 1.5 & 1.83 & 7.41 & 1.01 & 0.08 & 4.44 \\
\hline Apocynaceae & Rauyolfia pentaphyllaDucke & 7 & 1.3 & 1.6 & 3.7 & 0.51 & 0.05 & 3.07 \\
\hline Lauraceae & Persea americana Mill. & 7 & 1.3 & 1.6 & 3.7 & 0.51 & 0.05 & 3.06 \\
\hline Meliaceae & $\begin{array}{l}\text { Trichilia surinamensis (Miq.) } \\
\text { C. DC. }\end{array}$ & 6 & 1.1 & 1.37 & 11.11 & 1.52 & 0.1 & 4.98 \\
\hline Hernandiaceae & Hernandia guianensis Aubl. & 5 & 0.9 & 1.14 & 11.11 & 1.52 & 0.06 & 3.90 \\
\hline Anacardiaceae & Tapirira guianensis Aubl. & 5 & 0.9 & 1.14 & 11.11 & 1.52 & 0.02 & 3.12 \\
\hline Lauraceae & $\begin{array}{c}\text { Sextonia rubra (Mez) van } \\
\text { der Werff }\end{array}$ & 5 & 0.9 & 1.14 & 11.11 & 1.52 & 0.02 & 3.03 \\
\hline Fabaceae & $\begin{array}{l}\text { Hymenolobium } \\
\text { excelsumDucke }\end{array}$ & 4 & 0.7 & 0.92 & 11.11 & 1.52 & 0.33 & 9.28 \\
\hline Euphorbiaceae & $\begin{array}{l}\text { Hevea brasiliensis (Willd. ex } \\
\text { A. Juss.) Müll.Arg. }\end{array}$ & 4 & 0.7 & 0.92 & 7.41 & 1.01 & 0.12 & 4.47 \\
\hline Fabaceae & Ormosiasp & 4 & 0.7 & 0.92 & 7.41 & 1.01 & 0.09 & 3.88 \\
\hline Myristicaceae & $\begin{array}{l}\text { Virola melinonii (Benoist) } \\
\text { A.C. Sm. }\end{array}$ & 4 & 0.7 & 0.92 & 11.11 & 1.52 & 0.06 & 3.74 \\
\hline Myrtaceae & Eugenia prosoneura Berg. & 4 & 0.7 & 0.92 & 11.11 & 1.52 & 0.05 & 3.46 \\
\hline Malvaceae & Apeiba burchelliiSprague & 4 & 0.7 & 0.92 & 11.11 & 1.52 & 0.04 & 3.29 \\
\hline Humiriaceae & Saccoglottissp & 4 & 0.7 & 0.92 & 11.11 & 1.52 & 0.04 & 3.28 \\
\hline Vochysiaceae & $\begin{array}{l}\text { Salvertia convallariodora } \\
\text { St. Hill. }\end{array}$ & 4 & 0.7 & 0.92 & 14.81 & 2.02 & 0.01 & 3.21 \\
\hline Meliaceae & Carapa guianensis Aubl. & 4 & 0.7 & 0.92 & 11.11 & 1.52 & 0.02 & 2.83 \\
\hline Lecythidaceae & $\begin{array}{c}\text { Eschweilera odora (Poepp. ex } \\
\text { O. Berg) Miers }\end{array}$ & 4 & 0.7 & 0.92 & 11.11 & 1.52 & 0.02 & 2.79 \\
\hline
\end{tabular}


(Continua)

\begin{tabular}{|c|c|c|c|c|c|c|c|c|}
\hline Família & Espécies & NI & DA & DR & FA & FR & DoA & IVI \\
\hline Chrysobalanaceae & $\begin{array}{l}\text { HirtellabebecladaMoric. ex } \\
\text { DC. }\end{array}$ & 4 & 0.7 & 0.92 & 7.41 & 1.01 & 0.02 & 2.42 \\
\hline Annonaceae & Rollinia mucosa (Jacq.) Baill. & 4 & 0.7 & 0.92 & 3.7 & 0.51 & 0.01 & 1.68 \\
\hline Humiriaceae & Saccoglotis guianensis Benth. & 3 & 0.6 & 0.69 & 7.41 & 1.01 & 0.22 & 6.24 \\
\hline Caryocaraceae & $\begin{array}{l}\text { Caryocar villosum (Aubl.) } \\
\text { Pers. }\end{array}$ & 3 & 0.6 & 0.69 & 7.41 & 1.01 & 0.09 & 3.62 \\
\hline Fabaceae & Parkia multijugaBenth. & 3 & 0.6 & 0.69 & 7.41 & 1.01 & 0.06 & 2.92 \\
\hline Lecythidaceae & Lecythislurida (Miers) Mori & 3 & 0.6 & 0.69 & 7.41 & 1.01 & 0.05 & 2.63 \\
\hline Vochysiaceae & Qualea albifloraWarm. & 3 & 0.6 & 0.69 & 7.41 & 1.01 & 0.03 & 2.31 \\
\hline Melastomataceae & Miconiaprasina (S.W.) DC. & 3 & 0.6 & 0.69 & 7.41 & 1.01 & 0.02 & 2.01 \\
\hline Ochnaceae & $\begin{array}{l}\text { Ourateabexasperma (St. Hil.) } \\
\text { Baill. }\end{array}$ & 3 & 0.6 & 0.69 & 3.7 & 0.51 & 0.01 & 1.46 \\
\hline Olacaceae & Minquartia guianensis Aubl. & 2 & 0.4 & 0.46 & 7.41 & 1.01 & 0.1 & 3.48 \\
\hline Fabaceae & $\begin{array}{l}\text { Schizolobium amazonicum } \\
\text { Huber exDucke }\end{array}$ & 2 & 0.4 & 0.46 & 7.41 & 1.01 & 0.07 & 2.94 \\
\hline Fabaceae & $\begin{array}{l}\text { Clitoria racemosa } \\
\text { Sessé\&Moc. }\end{array}$ & 2 & 0.4 & 0.46 & 3.7 & 0.51 & 0.09 & 2.78 \\
\hline Annonaceae & Annona bypoglauca Mart. & 2 & 0.4 & 0.46 & 7.41 & 1.01 & 0.05 & 2.47 \\
\hline Rutaceae & $\begin{array}{l}\text { Zanthoxylum regnellianum } \\
\text { Engl. }\end{array}$ & 2 & 0.4 & 0.46 & 3.7 & 0.51 & 0.07 & 2.38 \\
\hline Myrtaceae & $\begin{array}{c}\text { Myrcia atramentifera Barb. } \\
\text { Rodr. }\end{array}$ & 2 & 0.4 & 0.46 & 7.41 & 1.01 & 0.03 & 2.08 \\
\hline Anacardiaceae & $\begin{array}{l}\text { Antrocaryon amazonicum } \\
\text { (Ducke) B.L. Burtt\& A.W. Hill }\end{array}$ & 2 & 0.4 & 0.46 & 7.41 & 1.01 & 0.02 & 1.98 \\
\hline Fabaceae & Hymenaea courbaril $\mathrm{L}$. & 2 & 0.4 & 0.46 & 3.7 & 0.51 & 0.02 & 1.46 \\
\hline Fabaceae & Andira parvifolia Benth. & 2 & 0.4 & 0.46 & 3.7 & 0.51 & 0.01 & 1.11 \\
\hline
\end{tabular}


(Conclusão)

\begin{tabular}{|c|c|c|c|c|c|c|c|c|}
\hline Família & Espécies & NI & DA & DR & FA & FR & DoA & IVI \\
\hline Goupiaceae & Goupia glabra Aubl. & 2 & 0.4 & 0.46 & 3.7 & 0.51 & 0.01 & 1.09 \\
\hline Chrysobalanaceae & Licania micrantha Miq. & 2 & 0.4 & 0.46 & 3.7 & 0.51 & 0.01 & 1.08 \\
\hline Fabaceae & Dinizia excelsa Ducke & 1 & 0.2 & 0.23 & 3.7 & 0.51 & 0.14 & 3.65 \\
\hline Moraceae & $\begin{array}{l}\text { Ficus adhatodifolia Schott } \\
\text { exSpreng. }\end{array}$ & 1 & 0.2 & 0.23 & 3.7 & 0.51 & 0.06 & 1.95 \\
\hline Euphorbiaceae & $\begin{array}{c}\text { Glycydendron } \\
\text { amazonicumDucke }\end{array}$ & 1 & 0.2 & 0.23 & 3.7 & 0.51 & 0.05 & 1.86 \\
\hline Malvaceae & Apeiba macropetalaDucke & 1 & 0.2 & 0.23 & 3.7 & 0.51 & 0.05 & 1.86 \\
\hline Euphorbiaceae & Hura crepitans $\mathrm{L}$. & 1 & 0.2 & 0.23 & 3.7 & 0.51 & 0.04 & 1.49 \\
\hline Clusiaceae & $\begin{array}{l}\text { Rheedia acuminata Miersex } \\
\text { Roca de Saldias, Yaneth }\end{array}$ & 1 & 0.2 & 0.23 & 3.7 & 0.51 & 0.02 & 1.13 \\
\hline Lauraceae & $\begin{array}{c}\text { Mezilaurus itauba (Meisn.) } \\
\text { Taub. exMez }\end{array}$ & 1 & 0.2 & 0.23 & 3.7 & 0.51 & 0.02 & 1.11 \\
\hline Calophyllaceae & $\begin{array}{c}\text { Calophyllum brasiliense } \\
\text { Cambess. }\end{array}$ & 1 & 0.2 & 0.23 & 3.7 & 0.51 & 0.01 & 1.01 \\
\hline Burseraceae & $\begin{array}{c}\text { Protium pubescens (Benth.) } \\
\text { Engl. }\end{array}$ & 1 & 0.2 & 0.23 & 3.7 & 0.51 & 0.01 & 0.96 \\
\hline Fabaceae & Peltogyne catingae Ducke & 1 & 0.2 & 0.23 & 3.7 & 0.51 & 0.01 & 0.96 \\
\hline Moraceae & Bagassa guianensis Aubl. & 1 & 0.2 & 0.23 & 3.7 & 0.51 & 0.01 & 0.93 \\
\hline Fabaceae & Vouacapoua americana Aubl. & 1 & 0.2 & 0.23 & 3.7 & 0.51 & 0.01 & 0.93 \\
\hline Malvaceae & Ceiba pentandra (L.) Gaertn. & 1 & 0.2 & 0.23 & 3.7 & 0.51 & 0.01 & 0.87 \\
\hline Rubiaceae & Genipa americana $\mathrm{L}$. & 1 & 0.2 & 0.23 & 3.7 & 0.51 & 0.01 & 0.85 \\
\hline Rubiaceae & Coussarea racemosa A. Rich. & 1 & 0.2 & 0.23 & 3.7 & 0.51 & 0 & 0.83 \\
\hline Sapotaceae & $\begin{array}{c}\text { Micropholis venulosa (Mart. } \\
\text { \&Eichler) Pierre }\end{array}$ & 1 & 0.2 & 0.23 & 3.7 & 0.51 & 0 & 0.82 \\
\hline Moraceae & Artocarpus beterophyllusLam. & 1 & 0.2 & 0.23 & 3.7 & 0.51 & 0 & 0.77 \\
\hline
\end{tabular}

Sendo: $\mathrm{NI}=$ Número de indivíduos; $\mathrm{DA}=$ Densidade absoluta, $\mathrm{DR}=$ Densidade relativa; $\mathrm{FA}=$ Frequência absoluta, $\mathrm{FR}=$ Frequência relativa; DoA= Dominância absoluta; IVI = Valor de importância. 
C. palmata, B. crassifólia, H. articulata, S. mombin, T. rboifolia e T. aurea foram as mais abundantes, representando $36,61 \%$ do total de indivíduos amostrados, $36,62 \%$ da densidade e $25,27 \%$ da frequência relativa, assim como apresentam $22,96 \%$ de valor de importância. 22,9\% das espécies apresentaram apenas um indivíduo, embora represente $3,9 \%$ dos indivíduos no total.

A dominância de C. palmata, B. crassifólia, H. articulata, T. rboifolia e $T$. aurea está de acordo com resultados encontrados na Amazônia, próximo às áreas vegetacionais naturais submetidas a forte pressão antrópica com completa ou parcial descaracterização da vegetação inicial (PINHEIRO et al., 2007; LAU; JARDIM, 2014).O surgimento de espécies lenhosas de rápido crescimento, como as encontradas neste estudo, possivelmente pode ter ligação aos impactos antrópicos nas proximidades das áreas amostradas. Segundo Jardim e Soares (2010), o adensamento das espécies oportunistas no primeiro estágio sucessional da vegetação nas clareiras é estimulada pela competição por nutrientes, água e luz com outras espécies.

Considerando a diversidade de uso pelos moradores da Resex rio Cajari, as principais espécies inventariadas que se destacam são C. guianensis, H. brasiliensis e D. odorata (Quadro 1).

Quadro 1. Principais espécies inventariadas segundo seus usos pelos moradores da Resex do rio Cajari, Amapá

(Continua)

\begin{tabular}{|c|c|c|c|}
\hline V.N & Espécie & Família & Usos \\
\hline Tatajuba & $\begin{array}{c}\text { Bagassa guianensis } \\
\text { Aubl. }\end{array}$ & Moraceae & $\begin{array}{c}\text { Alimentício, madeireiro, carpintaria, } \\
\text { dormentes, construçóes civil e naval, } \\
\text { marcenaria. }\end{array}$ \\
\hline Andiroba & $\begin{array}{c}\text { Carapa guianensis } \\
\text { Aubl. }\end{array}$ & Meliaceae & $\begin{array}{c}\text { Reumatismo, vermes, feridas e inflamações } \\
\text { da pele, cicatrizante, anti-inflamatório, } \\
\text { inflamação da garganta, repelentes de } \\
\text { insetos, micoses, construção civil e naval e } \\
\text { compensados. }\end{array}$ \\
\hline Piquia & $\begin{array}{c}\text { Caryocar villosum } \\
\text { (Aubl.) Pers. }\end{array}$ & Caryocaraceae & $\begin{array}{c}\text { Alimentação humana, alimentação animal, } \\
\text { construçóes civil e naval, compensados, } \\
\text { marcenaria, carpintaria, estacas. }\end{array}$ \\
\hline
\end{tabular}


(Conclusão)

\begin{tabular}{|c|c|c|c|}
\hline V.N & Espécie & Família & Usos \\
\hline Lixeira & Curatella americana $\mathrm{L}$. & Dilleniaceae & $\begin{array}{l}\text { Problemas do intestino e estômago, } \\
\text { diarreia, infecção vaginal e do útero, } \\
\text { lavagem de ferimentos, cicatrização de } \\
\text { feridas e reumatismo. }\end{array}$ \\
\hline Cumaru & $\begin{array}{l}\text { Dipteryx odorata } \\
\text { (Aubl.) Willd }\end{array}$ & Fabaceae & $\begin{array}{l}\text { Cosmético, o óleo no ramo alimentício, } \\
\text { veneno contra ratos, úlceras bucais, } \\
\text { coqueluche, dores de cabeça e das } \\
\text { articulações, trombose, tuberculose, } \\
\text { adenopatia, uso moveleiro e construção } \\
\text { civil. }\end{array}$ \\
\hline Seringueira & $\begin{array}{l}\text { Hevea brasiliensis } \\
\text { (Willd. ex A. Juss.) } \\
\text { Müll. Arg. }\end{array}$ & Euphorbiaceae & Indústria, alimentação animal e vermífugo. \\
\hline Itaúba & $\begin{array}{l}\text { Mezilaurus itauba } \\
\text { (Meisn.) Taub. ExMez }\end{array}$ & Lauraceae & $\begin{array}{l}\text { Construção naval e civil, carpintaria, } \\
\text { dormente, pranchas, tabuados, tacos. }\end{array}$ \\
\hline Tinteiro & $\begin{array}{c}\text { Miconia prasina }(\mathrm{Sw} .) \\
\text { DC. }\end{array}$ & Melastomataceae & $\begin{array}{l}\text { Madeira utilizada como combustível, } \\
\text { cabos de ferramentas. }\end{array}$ \\
\hline Barbatimão & $\begin{array}{l}\text { Ouratea bexasperma } \\
\text { (St. Hil.) Baill. }\end{array}$ & Ochnaceae & $\begin{array}{l}\text { Estanca hemorragias, infecção intestinal, } \\
\text { diarreia, lavagem de feridas, desinfecção } \\
\text { vaginal, garganta inflamada, gastrite, } \\
\text { problemas de estômago, energia e } \\
\text { alimentação animal. }\end{array}$ \\
\hline Tapereba & Spondias mombin $\mathrm{L}$. & Anacardiaceae & $\begin{array}{l}\text { Alimentação humana e animal, problemas } \\
\text { renais, diarreia, gripe, lavagem de } \\
\text { ferimentos, cicatrização de feridas, } \\
\text { inflamação uterina e vaginal, garganta } \\
\text { inflamada, tosse, banho de asseio pós- } \\
\text { parto. }\end{array}$ \\
\hline
\end{tabular}

Onde: N.V - Nome vernacular.

\subsection{FAMÍLIAS}

Dentre as famílias amostradas no Alto Cajari, as que apresentaram maior número de espécies foram: Fabaceae (15), Anacardiaceae (5), Lauraceae e Myrtaceae (4), Euphorbiaceae, Moraceae e Clusiaceae (3), correspondendo a 50\% do total. Das 34 famílias amostradas, 50\% estão representadas por apenas uma espécie (Figura 4). 


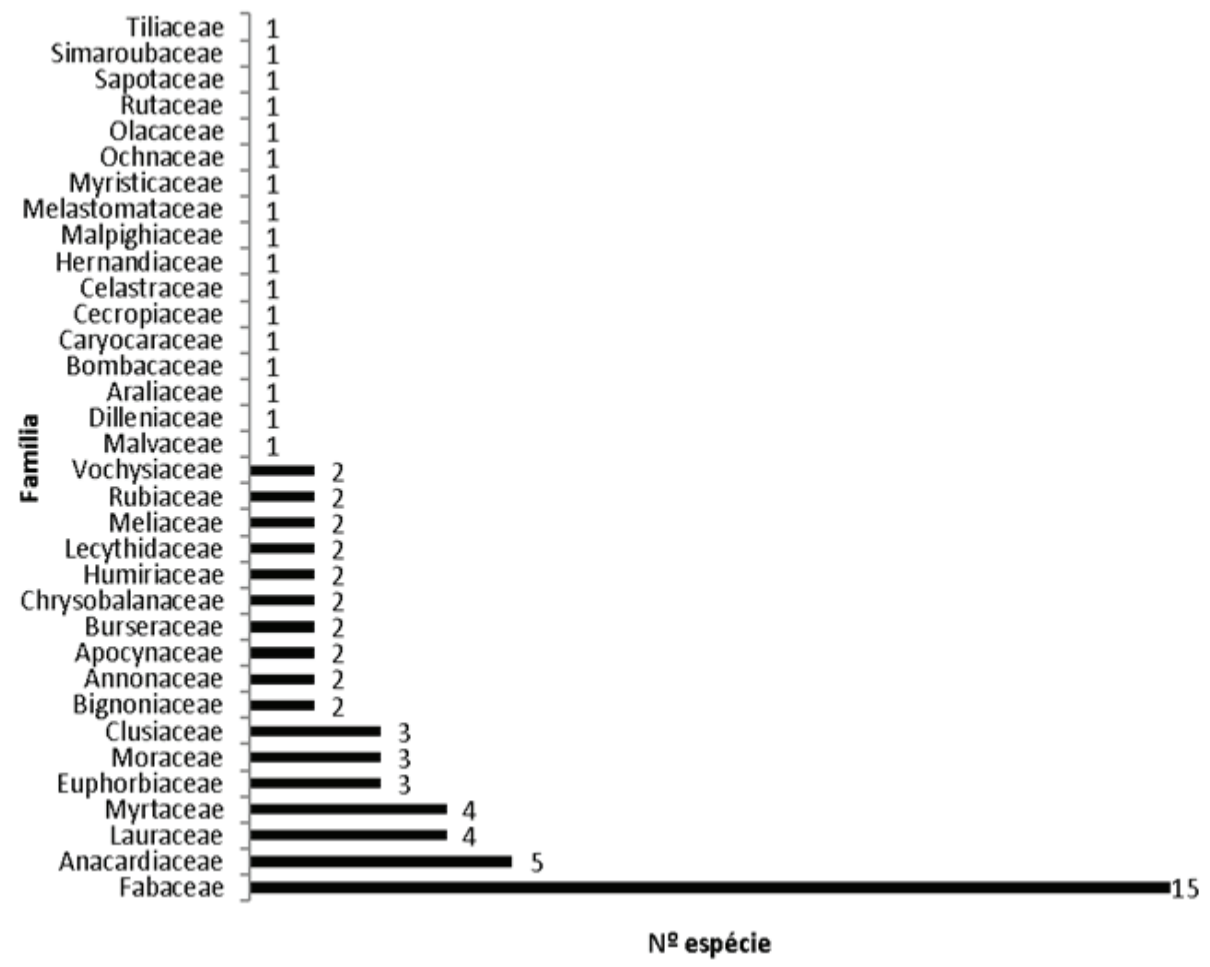

Figura 4. Distribuição crescente do número de espécies por famílias amostradas em 5,4 ha de vegetação no trecho Alto Cajari, Resex do rio Cajari, Amapá

Os resultados do estudo corroboram com os levantamentos florísticos realizados na Amazônia brasileira, os quais constataram a alta riqueza florística da família Fabaceae (OLIVEIRA, 1997; AMARAL; MATOS; LIMA, 2000; IVANAUSKAS; MONTEIRO; RODRIGUES, 2004; ARAUJO et al. 2009). Aparício (2011), ao estudar a estrutura da vegetação em diferentes ambientes na Resex do rio Cajari, constatou que a Fabaceae foi a que apresentou o maior número de espécies entre as famílias botânicas inventariadas. Os resultados corroboram também com Oestreich Filho (2014) em estudos em dois municípios do Mato Grosso.

A Figura 5 mostra a distribuição dos valores de importância para as famílias, destacando-se a Fabaceae $(59,76)$ e Anacardiaceae $(27,07)$. A primeira compreende-se ao fato de ocorrer em 19 das 27 parcelas amostradas, assim como apresentar indivíduos de porte relativamente alto e em maior número. Já a segunda 
corresponde ao fato de apresentar elevado número de indivíduos e relevantes diâmetros (contribuindo para o aumento da área basal), embora tenha baixo número de espécies.

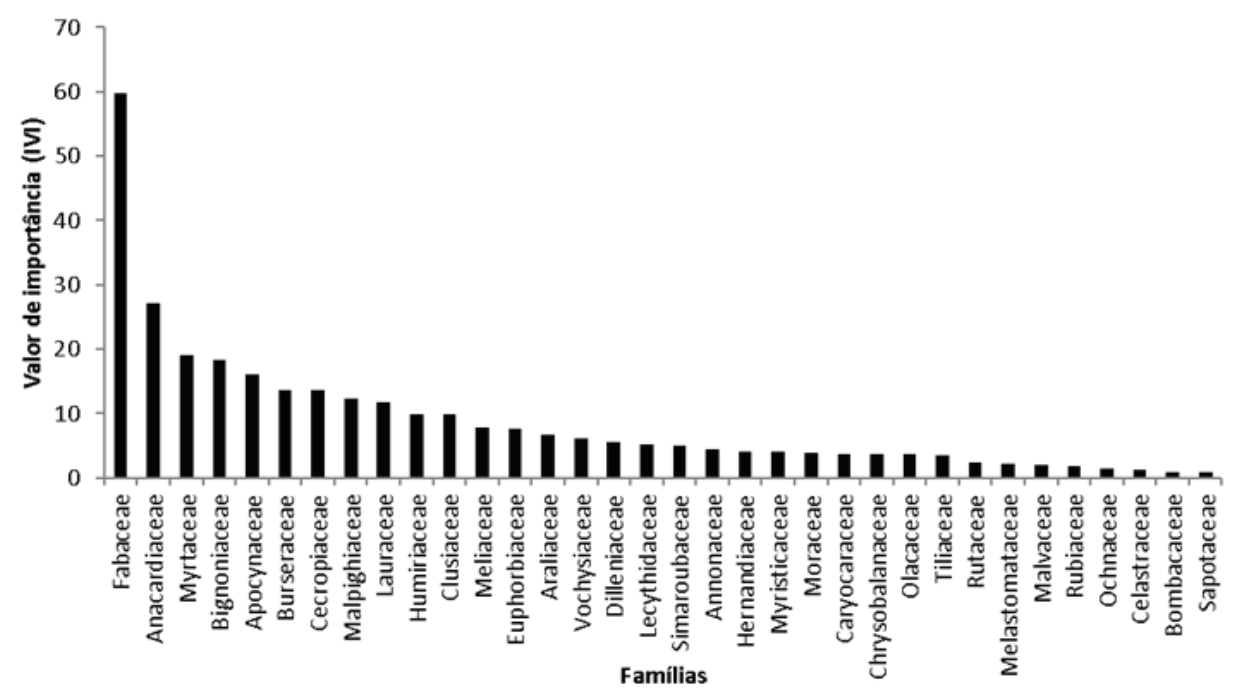

Figura 5. Distribuição decrescente do IVI para as famílias amostradas em 5,4 ha de vegetação no trecho Alto Cajari, Resex do rio Cajari, Amapá.

Algumas famílias muito diversas, como Myrtaceae e Bignoniaceae, perdem em importância por apresentar indivíduos de porte relativamente baixo, apesar do quantitativo de ocorrência ser alto, consequentemente baixa área basal total. Outras famílias com diversidade relativamente menor ou mesmo com poucas espécies, como Burseraceae e Cecropiaceae, acabam por ser mais importantes pelo grande número ou ao porte dos indivíduos. Os resultados corroboram com outros estudos florísticos na Amazônia (IVANAUSKAS; MONTEIRO; RODRIGUES, 2004; OLIVEIRA; AMARAL, 2004; KUNZ et al., 2008).

\subsection{DIVERSIDADE}

Os índices de diversidade, dominância e equabilidade apresentados na Tabela 3, mostram que a comunidade é altamente diversa. O valor do Índice de Morisita (Id) de 1,387 indica que ocorre alta proporção de indivíduos concentrados em poucas unidades amostrais, fazendo com que o padrão de distribuição seja agregado. 
Tabela 3. Índices de diversidade de Morisita (Id), Shannon (H') e Simpson (1-D), dominância de Simpson (D) e Equabilidade (E) para os 5,4 hectares amostrados no trecho Alto Cajari,Resex do rio Cajari, Amapá

\begin{tabular}{llllll}
\hline Índice & Id & $\mathrm{H}^{\prime}$ & $(1-\mathrm{D})$ & $\mathrm{D}$ & $\mathrm{E}$ \\
Valor & 1,387 & 3,874 & 0,975 & 0,025 & 0,9 \\
\hline
\end{tabular}

$\mathrm{O}$ valor do Índice de Shannon (H') igual a 3,87 indica que a comunidade amostrada apresenta uma distribuição mais uniforme do número de indivíduos em relação ao número de espécies. Quando baseado em dados empíricos, normalmente são encontrados valores entre 1,5 e 3,5 raramente ultrapassando 4,0 para logaritmo natural (nat) (MARGALEF, 1972).

Estudando a diversidade florística dos indivíduos com DAP $\geq 5 \mathrm{~cm}$, em fragmentos florestais de terra firme no município de Santana no Amapá, Freitas (2008), encontrou um valor de 3,59 para esse índice. A diversidade encontrada pelo índice de Shannon na área de estudo está dentro dos padrões encontrados em trabalhos realizados na Amazônia (RABELO et al., 2002; ROSA, 2002; FREITAS, 2008). Oestreich Filho (2014), estudando a florística de dois municípios no Mato Grosso, encontraram índices de Shannon de 3,34 e 3,56 para diferentes áreas de cerrado. Oliveira et al. (2015) encontraram 3,11 para uma área no município de Cruzeiro do Sul, Acre.

O índice de diversidade de Simpson $(1-D)$ de 0,975 também indica alta diversidade na população levantada. O índice de dominância de Simpson (D) indica que a comunidade não apresenta nenhuma dominância, apresentando valor de 0,025 , ou seja, sendo de $2,5 \%$ a probabilidade de amostrar dois indivíduos ao acaso que pertençam à mesma espécie.

A equabilidade também é muito alta, indicando que os valores de diversidade de Shannon são muito próximos ao máximo esperado para o número de espécies amostradas, demonstrando que as espécies contribuem com praticamente em igual número de indivíduos nas 27 parcelas inventariadas, ou seja, que as espécies contribuem com praticamente igual número de indivíduos na comunidade. Assim, os valores encontrados são próximos aos estudos fitossociológicos na Amazônia (MIRANDA, 2000; SALOMÃO; MATOS; ROSA, 2002; OLIVEIRA; AMARAL, 2004; ESPÍRITO-SANTO et al., 2005; MIRANDA; ALMEIDA; DANTAS, 2006; ALARCÓN; 
PEIXOTO, 2007; ALVES; MIRANDA, 2008; FERREIRAJÚNIOR et al., 2008; GONÇALVES; SANTOS, 2008; OESTREICH FILHO, 2014; OLIVEIRA et al., 2015).

\section{CONSIDERAÇÕES FINAIS}

A área amostrada apresentou relevante riqueza de espécies e equilíbrio em suas distribuições, principalmente por possuir baixo número de espécies "localmente raras", o que beneficiou sua composição florística arbórea.

As famílias Fabaceae e Anacardiaceae foram as que apresentaram maior riqueza de espécies e número de indivíduos, assim como os maiores valores de importância. As espécies C. palmata, B. crassifolia e $H$. articulata apresentaram os maiores números de ocorrência, indicando antropização na área.

A distribuição diamétrica seguiu o padrão para florestas tropicais, demonstrando relação positiva entre recrutamento e mortalidade de indivíduos, essencial para o balanço estrutural do componente arbóreo.

Apesar de os resultados, a localização da área (circunvizinha à rodovia) e seu fácil acesso, bem como o fato da Resex rio Cajari não possuir plano de manejo são elementos preocupantes para a manutenção da diversidade florística e gestão da unidade de conservação.

\section{REFERÊNCIAS}

ALARCÓN, J. G. S.; PEIXOTO, A. L. Florística e fitossociologia de um trecho de um hectare de floresta de terra firme, em Caracaraí, Roraima, Brasil. Boletim Museu Paraense Emílio Goeldi. Ciências Naturais, Belém, v. 2, n. 2, p. 33-60, 2007.

ALVES, A. I. Descrição da reserva extrativista do rio Cajarí. In: MURRIETA, J.R.; RUEDA, M.P. União Mundial para a Conservação da Natureza-UICN: reservas extrativistas. Reino Unido: [s.n.], 1995.

ALVES, J. C. Z. O.; MIRANDA, I. S. Análise da estrutura de comunidades arbóreas de uma floresta amazônica de Terra Firme aplicada ao manejo florestal. Acta amazônica, 
v. 38, n. 4, p. 657-666, 2008.

AMARAL, I. L.; MATOS, F. D. A.; LIMA, J. Composição florística e parâmetros estruturais de um hectare de floresta densa de terra firme no Rio Uatumã, Amazônia, Brasil. Acta Amazonica, v. 30, n. 3, p. 377-392. 2000.

APARÍCIO, W. C. S. Estrutura da vegetação em diferentes ambientes na Resex do Rio Cajari: interações solo-floresta e relações com a produção de castanha. 2011. 150f. Tese (Doutorado) - Universidade Federal Rural de Pernambuco, Recife, PE.

APG III. An update of the Angiosperm Phylogeny Group classification for the orders and families of flowering plants: APG III. Botanical JournaloftheLinneanSociety, v. 161, n. 2, p. 105-121, 2009.

ARAUJO, R. A.; COSTA, R. B.; FELFILI, J. M.; GONÇALVES, I. K.; SOUSA, R. A. T. M.; DORVAL, A. Florística e estrutura de fragmento florestal em área de transição na Amazônia Matogrossense no município de Sinop. Acta Amazonica, v. 39, n. 4, p. 865-878, 2009.

BENJAMIN, A. M. S. Agroextrativismo: sustentabilidade e estratégias produtivas na reserva extrativista do rio Cajarí, sul do Amapá. 2004. 135f. Dissertação (Mestrado) Universidade Federal doPará, Belém, PA.

BRUMMITT, R.K.; POWELL, C.E. Authors of plant names: a list of authors of scientific names of plants, with recommended standard forms of their names, including abbreviations. England: Royal BotanicGardens, Kew, 1992.

DRUMmOND, J. A.; DIAS, T. C. A. C.; BRITO, D. M. C. Atlas das unidades de conservação do Estado do Amapá. Macapá: MMA/IBAMA-AP/GEA/SEMA, 2008.

ESPÍRITO-SANTO, F. D. B.; SHIMABUKURO, Y. E.; ARAGÃO, L. E. O. C.; MACHADO, E. L. M. Análise da composição florística e fitossociológica da floresta nacional do Tapajós com o apoio geográfico de imagens de satélites. Acta amazônica, v. 35, n. 2, p. 155-173, 2005.

ESQUERDO, P. A.; MARQUES, R. G.; RANGEL, K.S. A região do Rio Cajari e sua 
produção de borracha no século XX. In: ENCONTRO NACIONAL DE GEÓGRAFOS, 18., 2016, São Luiz. Anais... São Luiz: IFMA - MA, 2016.

FERREIRA JÚNIOR, E. V.; SOARES, T. S.; COSTA, M. F. F.; SILVA, E. S. M. Composição, diversidade e similaridade florística de uma floresta tropical semi decídua sub montana em Marcelândia - MT. Acta amazônica, v. 38, n. 4, p. 673-680, 2008.

FLORA DO BRASIL 2020 EM CONSTRUÇÃO. Flora do Brasil 2020. Jardim Botânico do Rio de Janeiro. Disponível em: < http://floradobrasil.jbrj.gov.br/> Acesso em: 12 Dez. 2016.

FREITAS, J. L. Sistemas Agroflorestais e sua utilização como instrumento de uso da terra: o caso dos pequenos agricultores da Ilha de Santana, Amapá, Brasil. 2008. 244f. Tese (Doutorado) - Universidade Federal Rural da Amazônia e Embrapa, Amazônia Oriental, Belém, PA.

FREITAS, J. L.; SANTOS, E. S.; SILVA, R. B. L.; SILVA, T. L. Composição florística de fragmentos florestais para arranjo em sistemas agroflorestais, na Ilha de Santana, Amapá. In: SIMPÓSIO LATINO-AMERICANO SOBRE MANEJO FLORESTAL, 5., 2011, Santa Maria. Anais... 2011.

GONÇALVES, F. G.; SANTOS, J. R. Composição florística e estrutura de uma unidade de manejo florestal sustentável na Floresta Nacional do Tapajós, Pará. Acta amazônica, v. 38, n. 2, p. 229-244, 2008.

GOVERNO DO ESTADO DO AMAPÁ - GEA. Plano de prevenção e controle do desmatamento e queimadas do Estado do Amapá - PPCDAP: contexto e ações. Macapá, 2009. 99f.

HIGUCHI, N.; SANTOS, J.; JARDIM, F.C.S. Tamanho de parcelas amostrais para inventários florestais. Acta Amazonica, v. 12, n. 1, p. 91-103, 1982.

IVANAUSKAS, N. M.; MONTEIRO, R.; RODRIGUES, R. R. Estrutura de um trecho de floresta Amazônica na bacia do alto rio Xingu. Acta amazônica, v. 34, n. 2, p. 281305, 2004. 
JARDIM, F.C.S. Comportamento da regeneração natural de espécies arbóreas em diferentes intensidades de desbastes por anelamento, na região de Manaus - AM. 1995. 162f. Tese (Doutorado) - Universidade Federal de Viçosa, 1995.

JARDIM, F.C.S.; SOARES, M.S. Comportamento de SterculiaPruriens (Albl.) Schum. em floresta tropical manejada em Moju-PA. Acta Amazonica, Manaus, v. 40, n.3, p. 535-542, 2010.

KEW MONOCOT WORLD CHECKLIST. Orchidaceae. Disponível em: <www.kew. org/wcsp > . Acesso em: 22 out. 2012.

KUNZ, S. H.; IVANAUSKAS, N. M.; MARTINS, S. V.; SILVA, E.; STEFANELLO, D. Aspectos florísticos e fitossociológicos de um trecho de Floresta Estacional Perenifólia na Fazenda Trairão, Bacia do rio das Pacas, Querência-MT. Acta amazônica, v. 38, n. 2, p. 245-254, 2008.

LAU, A. V.; JARDIM, M. A. G. Composição e densidade do banco de sementes em uma floresta de várzea, Ilha do Combu, Belém-PA, Brasil. Biota Amazônia, v. 4, n. 3, p. 5-14, 2014.

MARGALEF, R. Homage to Everlyn Hutchinson, or why there an upper limit to diversity. Transactions Connecticult Academy of Arts and Sciences v. 44, p. 211235, 1972.

MINISTÉRIO DO MEIO AMBIENTE - MMA. Amazônia. Disponível em: < http:// www.mma.gov.br/biomas/amazônia > . Acesso em: 12 dez. 2016.

MIRANDA, I. S. Análise florística e estrutural da vegetação lenhosa do rio Comemoração, Pimenta Bueno, Rondônia, Braisl. Acta amazônica, v. 30, n. 3, p. 393-422, 2000.

MIRANDA, I. S.; ALMEIDA, S. S.; DANTAS, P. J. Florística e estrutura de comunidades arbóreas em cerrados de Rondônia, Brasil. Acta amazônica, v. 36, n. 4, p. 419-430, 2006.

MISSOURI BOTANICAL GARDEN TROPICOS. Plant Tropics. Disponível em: < http:// 
www.tropicos.org > . Acessoem: 30 set. 2015.

MORA, C.; TITTENSOR D. P; SINA, A.; SIMPSON, A. G. B.; WORM, B. 2011.How Many Species Are There on Earth and in the Ocean? PlosBiology, v. 9, n. 8, p. e1001127. DOI:10.1371/journal.pbio.1001127.

MUELLER-DOMBOIS, D.; ELLENBERG, H. Aims and methods of vegetation ecology. New York: John Wiley\& Sons, 1974.

OESTREICH FILHO, E. Fitossociologia, diversidade e similaridade entre fragmentos de cerrado stricto sensu sobre neossolosquartzarênicosórticos, nos municípios de Cuiabá e Chapada dos Guimarães, estado de Mato Grosso, Brasil. 2014. 88f. Dissertação (Mestrado em Ciências Florestais e Ambientais) Universidade Federal de Mato Grosso, 2014.

OLIVEIRA, A. A. Diversidade, estrutura e dinâmica do componente arbóreo de uma floresta de terra firme de Manaus, Amazonas. 1997 199f. Tese (Doutorado) - Universidade de São Paulo, São Paulo.

OLIVEIRA, A. N.; AMARAL, I. L. Florística e fitossociologia de uma floresta de vertente na Amazônia Central, Amazonas, Brasil. Acta amazonica, v. 34, n. 1, p. 21-34, 2004.

OLIVEIRA, E. K. B.; NAGY, A. C. G.; BARROS, Q. S.; MARTINS, B. C.; MURTA JÚNIOR, L. S. Composição florística e fitossociológica de fragmento florestal no sudoeste da Amazônia. Enciclopédia Biosfera, v. 11 n. 21, p. 21-26, 2015.

PELLICO NETO, S.; BRENA, D.A. Inventário florestal. Curitiba: UFPR. 1993.

PINHEIRO, K. A. O.; CARVALHO, J. O. P.; QUANZ, B.; FRANCEZ, L. M. B.; SCHWARTZ, G. Fitossociologia de uma área de preservação permanente no leste da Amazônia: indicação de espécies para recuperação de áreas alteradas. Floresta, v. 37, n. 2, p. 175-187, maio/ago. 2007.

QUEIROZ, W.T. Técnicas de amostragem em inventário florestal nos trópicos. Belém: FCAP. 1998. 
RABELO, F.G.; ZARIN, D.J.; OLIVEIRA, F.A.; JARDIM, F.C.S. Diversidade, composição florística e distribuição diamétrica do povoamento com $\mathrm{DAP} \geq 5 \mathrm{~cm}$ em região do estuário do Amapá. Revista de Ciências Agrárias, Belém, v. 37, n. 37, p. 91-112, 2002.

ROSA, L. S. Limites e possibilidades do uso sustentável dos produtos madeireiros e não madeireiros na Amazônia brasileira: o caso dos pequenos agricultores da Vila Boa Esperança, em Moju, no Estado do Pará. Belém. 2002. 304f. Tese (Doutorado) - Universidade Federal do Pará, Núcleo de Altos Estudos da Amazônia, AM.

SALOMÃO, R. P.; MATOS, A. H.; ROSA, N. S. Dinâmica do sub-bosque e do estado arbóreo de floresta tropical primária fragmentada na Amazônia Oriental. Acta amazônica, v. 32, n. 3, p. 387-419, 2002.

SANTOS, E. S.; APARÍCIO, P. S.; SILVA, T. L.; FREITAS, J. L. Estrutura da espécie Virola surinamensis (Rol.) Ward na Floresta Estadual do Amapá- FLOTA/AP. Revista de Biologia e Ciências da Terra, v. 13, n.1, p. 48-61, 2013.

SANTOS, G. C.; JARDIM, M. A. G. Florística e estrutura do estrato arbóreo de uma floresta de várzea no município de Santa Bárbara do Pará, Estado do Pará, Brasil. Acta amazônica, v. 36, n. 4, p. 437-446, 2006.

SHEPHERD, G. J. FITOPAC 1: manual do usuário. Campinas: Universidade Estadual de Campinas. 1995. 78 p.

SISTEMA DE INFORMAÇÃO SOBRE A BIODIVERSIDADE BRASILEIRA SiBBr. Biodiversidade brasileira. Disponível em: <http://www.sibbr.gov.br/ areas/?area = biodiversidade $>$. Acesso em: 12 dez. 2016.

WILSON, E. O. A Situação atual da diversidade biológica em Biodiversidade. Rio de Janeiro, Nova Fronteira, 657 p. 1997

Recebido em: 2016-03-08 Aceito em: 2017-02-13 